\title{
Zur aktuellen Situation der Innovationsfinanzierung in Deutschland
}

\author{
Lothar Brunsch
}

\section{Vorbemerkungen}

Nach langen Jahren einer verhaltenen bis zurïckhaltenden Diskussion und Handhabung der Probleme des technologieorientierten Gründungsgeschehens, der Wege zur Verbesserung der Innovationsfinanzierung bei kleinen und mittleren Unternehmen und der Gestaltung des Beteiligungskapitalmarktes in Deutschland kann heute von einer Trendwende bis hin zum wahren Boom auf diesem Gebiet gesprochen werden.

Mit dieser Entwicklung werden das Gründungsgeschehen und die Entwicklung von kleinen und mittleren Unternehmen management- und kapitalseitig wirksam unterstuitzt. Wenn davon auszugehen ist, dass das Gründungsgeschehen von heute die Zukunft des deutschen Wirtschaftsstandortes von morgen, d. h. die Lebensqualität der Bevölkerung entscheidend mit beeinflusst, dann sind viele gefordert, besonders die Hochschulen, die viel für ein gründerfreundliches Klima leisten können. Hierzu gehört auch die verstärkte Umsetzung der Hochschulforschungsergebnisse in marktfähige Produkte und Dienstleistungen oder die Realisierung von Geschäftsideen, die in bestehenden Unternehmen nicht weiter verfolgt werden.[1]

\section{Zielgruppen der Innovationsfinanzierung}

\subsection{Innovierende Unternehmen}

Die vorliegenden Untersuchungsergebnisse machen deutlich, dass es um zwei Gruppen von Unternehmen geht, auf die sich die Innovationsfinanzierung bezieht. Häufig wird die Innovationsfinanzierung nur auf neue Technologieunternehmen und Risikokapital orientiert. Dabei wird außer Acht gelassen, dass die bestehenden kleinen und mittleren Unternehmen ebenfalls beträchtliche Innovationsanstrengungen unternehmen; denn nur so können sie ihre Marktstellung behaupten oder ausbauen. Diese innovierenden Unternehmen ,stellen eine äußerst heterogene Gruppe nach Größe, Alter, wirtschaftlicher Unabhängigkeit, Branchenzugehörigkeit, Technologiebereichen sowie vor allem auch nach Regelmäßigkeit und Schwerpunkten ihrer FuE-Tätigkeit dar."[2] Gerade diese Merkmale beeinflussen die einzelnen Finanzierungsquellen sehr stark. Die innovierenden Unternehmen haben meist eine deutlich höhere FuE-Intensität als die Großunternehmen mit einer großen Streubreite. 1996 haben nach Schätzungen des BMBT diese Unternehmen ca. 1,7 Mrd. DM für FuE direkt ausgegeben plus der Aufwendungen für die Markteinfuihrung bzw. Ver- marktung der Dienstleistungen (noch einmal bis 50 \%). Solche Unternehmen finanzieren ihre Innovationsprojekte vor allem aus eigenen Mitteln (vgl. Abbildung 1).

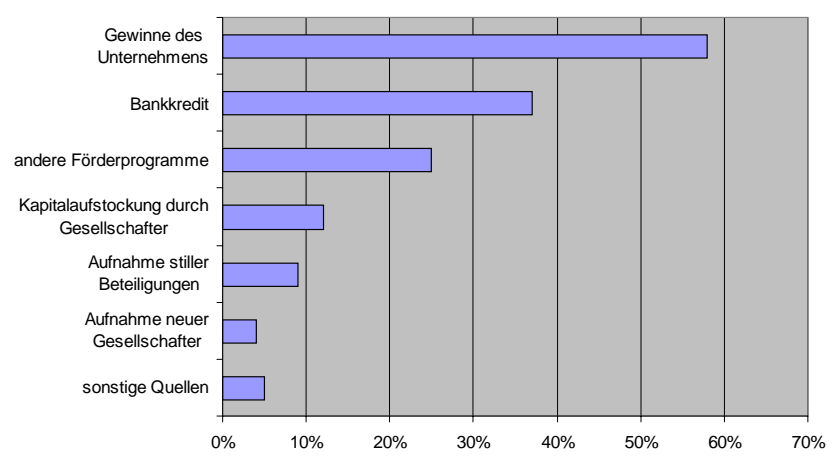

Abb. 1: Finanzierungsquellen für Innovationsprojekte von KMU [3]

Gleichzeitig weisen Untersuchungen auf folgende Hemmnisse für die FuE-Tätigkeit dieser Unternehmen hin (vgl. Abbildung 2).

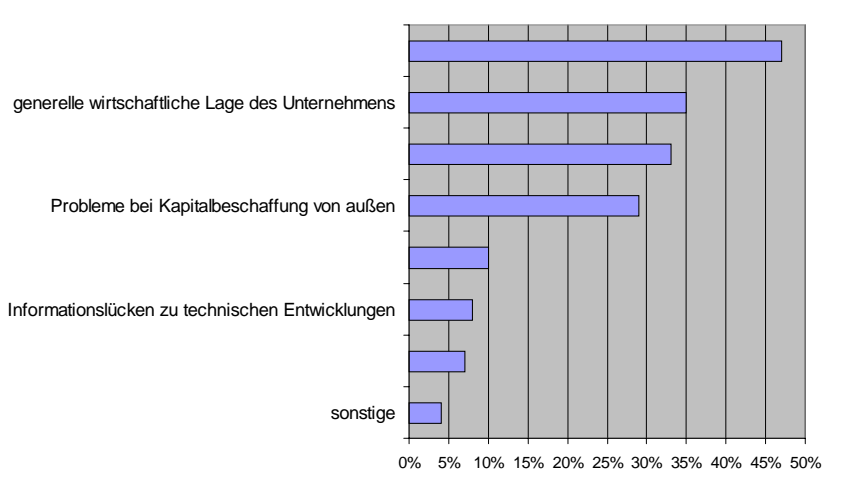

Abb. 2: Hemmnisse für die FuE-Tätigkeit [4]

Für die umfangreichen FuE-Arbeiten reicht häufig, wie die Abbildung 2 zeigt, die Selbstfinanzierung der innovierenden Unternehmen nicht aus. Sie benötigen des weiteren verschiedene verfuigbare Quellen der Außenfinanzierung.

\subsection{Besonderheiten der jungen Technologieunter- nehmen (JTU)}

Die Besonderheiten der jungen Technologieunternehmen bestehen vor allem darin, dass sie noch geringere Möglichkeiten der Selbstfinanzierung haben. Deshalb ist für sie die Außenfinanzierung von noch größerer Bedeutung. Im Gegensatz zu den bereits etablierten KMU suchen die JTU in der Frühphasenfinanzierung meist fuir ein einziges Innovationsprojekt Kapital von außen. Die weitere Geschäftsentwicklung des gesamten Unterneh- 
mens hängt demzufolge vom Erfolg dieses Projektes ab.

„JTU bringen aus Sicht eines klassischen Kapitalgebers insgesamt weitaus ungünstigere Finanzierungsbedingungen mit sich als etablierte Unternehmen:

- Risiken technischer, marktseitiger sowie sonstiger Art durch fehlende Marktetablierung und Organisationskonsolidierung, Abhängigkeit des Geschäftsverlaufs von einem einzigen Innovationsprojekt, keine unternehmensinterne Risikostreuung,

- ungenau abschätzbarer und bei anspruchsvollen Innovationsvorhaben vergleichsweise hoher Kapitalbedarf,

- oft Notwendigkeit von Nachfinanzierungen; effektiver Kapitalbedarf erfahrungsgemäß deutlich über anfänglich prognostizierten Werten, zudem langer Zeitraum der Mittelbindung,

- Fehlen dinglicher Sicherheiten oder nur Sicherheiten mit niedrigem Beleihungswert,

- hoher Beratungsbedarf, daher kostenintensiver Unterstiitzungsaufwand für Kapitalgeber,

- bei Nachfrage geringer Kapitalbeträge ungünstige Relation von Ausfallrisiken und Unterstuitzungsaufwand zu Entgelten oder Wertsteigerungen.

Führt man sich diese Besonderheiten von JTU aus Sicht eines Kapitalgebers vor Augen, wird verständlich, dass viele Bereiche der traditionellen Unternehmensfinanzierung für sie nur schwer erschließbar sind. Vor allem besteht für die an Sicherheiten orientierte Kreditfinanzierung kein großer Spielraum für die frühe Aufbauphase."[5]

\section{Aktuelle Finanzierungsquellen der innovierenden KMU und JTU}

\subsection{Innen- und Außenfinanzierung als Finanzierungsquellen}

Grundsätzlich kann die Kapitalbeschaffung aller innovierenden Unternehmen und JTU's von innen oder außen erfolgen (vgl. Abbildung 3).

Bei der Innenfinanzierung ist zwischen der Selbstfinanzierung (aus zurüickbehaltenen Gewinnen), der Finanzie- rung aus Rückstellungen, der Finanzierung aus Abschreibungsrüickflüssen und der Finanzierung aus freigesetztem Kapital (Vermögensumschichtung) zu unterscheiden. Bei der Außenfinanzierung erhält das Unternehmen Kapital direkt von Einzelpersonen, resp. von Banken oder über den Geld- bzw. Kapitalmarkt. Für die innovierenden Unternehmen sind die typischen Finanzierungsformen:

- Innenfinanzierung mit allen genannten Möglichkeiten, je nach den betrieblichen Gegebenheiten;

- Außenfinanzierung in Form von:

Fremdkapital (von Banken, anderen Unternehmen, Privatpersonen),

Öffentliche Förderung (Zuschüsse, Darlehen und u. U. auch Bürgschaften),

Lieferantenkredite (einschl. Kundenanzahlungen)

Börsenemission,

Beteiligungskapital.

Die wesentlichen Finanzierungsquellen für JTU sind:

- Eigenmittel der Gründer, die häufig den geringeren Anteil am Eigenkapital ausmachen;

- öffentliche Programme (Förderung von Innovationsvorhaben oder einzelner Aktivitäten im Innovationsprozess von KMU (z. B. FuE-Kooperationen), Förderung des Aufbaus von Existenzgründungen generell oder einzelner Schritte im Aufbauprozess einer Neugründung (z. B. Beratungshilfen), Förderung von Investitionen in mittelständischen Unternehmen);

- Beteiligungsgesellschaften (mit dem Ziel der Wirtschaftsförderung von KMU sowie neuer Unternehmen oder renditeorientierter Gesellschaften bzw. Business Angels);

- Innenfinanzierung (Vermarktung von Dienstleistungen, erste Erlöse aus Entwicklungsprojekten);

- Fremdkapital (Kontokorrentlinien, in geringerem Umfang auch langfristige Darlehen, soweit durch die Gesellschafter oder Dritte (z. B. öffentliche Bürgschaften) besichert).

Studien des ISI (Fraunhofer Institut Systemtechnik und Innovationsforschung) zu geförderten JTU zeigen, dass deren Kapitalbedarf bis zur Marktetablierung i. d. R. so hoch ist, dass er die finanziellen Ressourcen der Gründer bei weitem übersteigt. Er liegt häufig in einer Größenord-

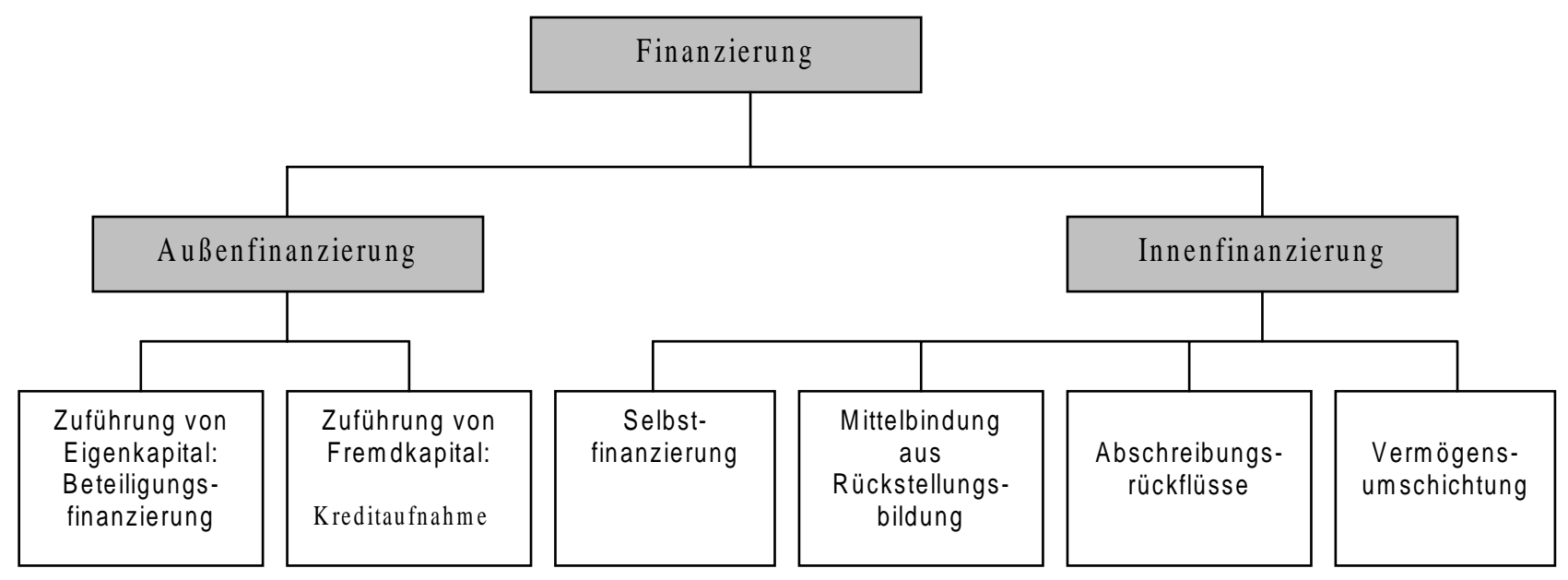

Abb. 3: Finanzierungsquellen eines Unternehmens 
nung von 1 bis 2 Mio. DM, jeweils für die Produktentwicklungs- und für die Markteinführungsphase. Für solche JTU mit einem besonders hohen Wachstum bewegt sich der Bedarf im Bereich von 5 bis 10 Mio. DM für die erste Finanzierungsrunde und für weitere Runden noch deutlich dariuber.“ (vgl. Abbildung 4) [6]

Abb. 4: Finanzierungsmöglichkeiten in den Unternehmensphasen

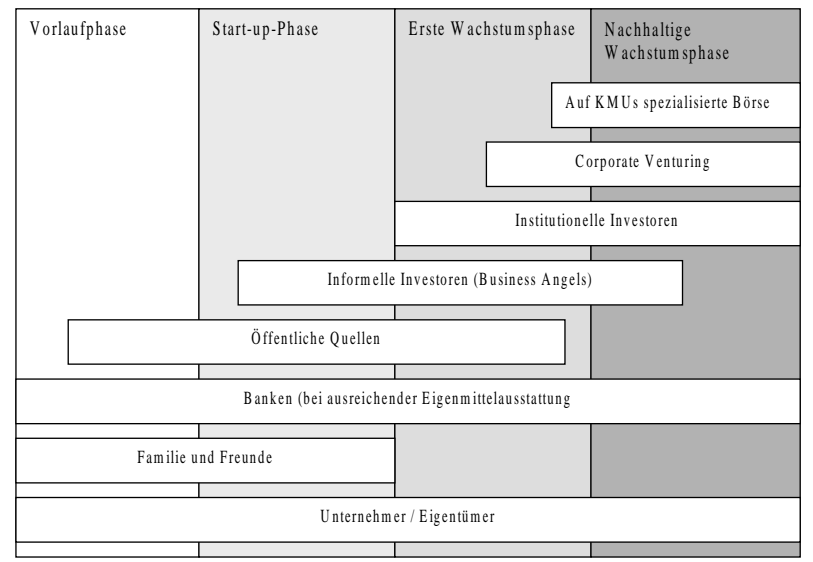

In den folgenden Abschnitten werden die aktuellen Möglichkeiten und Grenzen für innovierende Unternehmen und JTU ausfuihrlicher behandelt.

\subsection{Aktuelle Situation der Innovationsfinanzierung nach Finanzierungsquellen}

\subsubsection{Innenfinanzierung}

Die Innenfinanzierungsmöglichkeiten zur Deckung der FuE-Aufwendungen werden maßgeblich von der Ertragskraft der Unternehmen bestimmt. Demzufolge trifft diese Finanzierungsform vor allem für innovierende Unternehmen zu. Die Innenfinanzierungsmöglichkeiten werden auch stark bestimmt von der Unternehmensbesteuerung. Die Innenfinanzierung hat für die JTU's, wie bereits oben erwähnt, nur sehr begrenzte Möglichkeiten. Für sie hängt es davon ab, wie schnell es die Unternehmen schaffen, über die Umsätze ausreichende Deckungsbeiträge zu erzielen. Die Dekkungsbeiträge können aus eigenständigen Dienstleistungsgeschäften oder aus der Vermarktung von Teilergebnissen des eigentlichen Entwicklungsprojektes erwirtschaftet werden. Beides führt rasch zur Stärkung der Innenfinanzierung, birgt aber auch folgende Gefahren in sich:

- Das eigentliche innovative Produkt oder Dienstleistungsgeschäft wird nicht konsequent genug verfolgt. Das kann zu Zeitverlusten im Kerngeschäft führen.

- Die knappen Ressourcen werden verzettelt eingesetzt.

- Das Unternehmen verliert sein Innovationsimage.

Für JTU mit ungeeigneter Kostenkontrolle, ineffizienter Organisation und geringen Gewinnen wird die Innenfinanzierung rasch Grenzen für den Aufbauprozess setzen und damit die Möglichkeiten der Außenfinanzierung beeinflussen.

\subsubsection{Außenfinanzierungsquellen}

a) Fremdkapital von Banken

Da es keinen spezifischen Innovationskredit gibt, kann dessen Rolle im Firmenkundengeschäft nur geschätzt werden. Nach Untersuchungen von Kulicke/Roß/Grundrum von 1997 geben Banken höchstens 3 \% ihrer Kredite in Form von Innovationskrediten aus. Die Innovationskredite haben wenig mit dem klassischen Aktivgeschäft der Bank zu tun, da hier in der Regel keine realen Sicherheiten vorliegen. Die Kreditwürdigkeitsprüfung erfordert vor allem eine Prüfung der technologischen Realisierbarkeit, der Marktchancen einschließlich der sich daraus ergebenden Folgen für die Vertragsaussichten. Es gilt hier nicht fuir die Banken, wie allgemein üblich, die Vergangenheit zu analysieren und die Bewertung der Sicherheiten vorzunehmen, sondern es werden Bankmitarbeiter benötigt, die eine technologische (innovative) Bonitätsprüfung realisieren können. Das Hauptproblem ist dabei immer noch die asymmetrische Informationsverteilung zwischen kapitalsuchenden Unternehmen und den Banken und das Fehlen werthaltiger Sicherheiten; deshalb unterbleibt häufig eine Kreditfinanzierung. Bis auf zwei Änderungen gibt es hier nichts Neues zu berichten.

„Lediglich in folgenden zwei Bereichen sind Änderungen eingetreten:

- Der Bund hat seit Anfang der 90er Jahre den Kreditsektor stärker in die Förderung marktnaher FuE-Tätigkeiten eingebunden (zunächst über das FuE-Darlehens- und das KfW-Innovationsprogramm, jetzt über das ERP-Innovationsprogramm). Doch bezieht sich diese Integration nicht auf die eigentliche Finanzierungsfunktion des Kreditsektors, sondern er leitet die Kredite durch und übernimmt gegenüber dem Fördergeber bzw. der KfW die volle oder teilweise Primärhaftung (Förderabwicklung im Hausbankenverfahren). Die Förderdarlehen müssen von den Darlehensnehmern banküblich abgesichert werden. Mit der begrenzten Haftungsfreistellung für die durchleitende Bank soll dieser ein größerer Spielraum bei der Bewertung der Sicherheiten geboten werden.

- Seit Ende 1995 gibt es Ansätze im Kreditsektor, die fehlende interne Kompetenz zur Bewertung der technischen und Marktseite eines Innovationsvorhabens von KMU bei Kredit- oder Beteiligungsentscheidungen durch Einbindung externer Fachleute aus Forschungseinrichtungen und Hochschulen auszugleichen. Auf diese Weise soll die Entscheidungsbasis von Firmenkundenberatern in den Instituten der Sparkassenorganisation deutlich verbessert werden."[7]

Die öffentlichen Programme haben die Bereitschaft der Banken erhöht, Kredite für JTU's bereitzustellen. Trotzdem bleibt die Finanzierungsquelle Kredit für die JTU in den ersten Geschäftsjahren, sofern keine banküblichen Sicherheiten vorliegen, sehr begrenzt und sie beschränken sich auf Kreditlinien im kurzfristigen Bereich. Positiv auf die Kreditvergabe durch die Banken wirkt sich das Engagement der Business Angel (BA) im Unternehmen aus. 


\section{b) FuE-Förderung}

Nach Angaben des Stifterverbandes der Wissenschaftsstatistik trug 1995 die Wirtschaft der Alten Bundesländer (ABL) selbst $90 \%$ der Forschungsleistungen. 1985 waren es nur $86 \%$. Auf Grund der strengen EU-Richtlinien ist eine Förderung nach der FuE-Phase nur sehr beschränkt möglich. Für die Förderung der FuE in KMU standen 1995 1,2 Mrd. DM zur Verfügung. Darin enthalten sind auch Leistungen, die nicht selbst von den KMU erbracht werden, ihnen aber dienen, wie z. B. Patentauslegestellen oder Zentren für Informationen und Beratungen. Fast zwei Drittel der Fördermittel kamen in der gewerblichen Wirtschaft der Neuen Bundesländer (NBL) zum Einsatz. Vom Programm des Zuständigkeitsbereiches des Bundesministeriums für Bildung, Wissenschaft, Forschung und Technologie (BMWi) waren es $76 \%$. Die einzelnen Bundesländer besitzen eine Vielzahl von Maßnahmen im Bereich der einzelbetrieblichen Innovationsförderung und des Technologietransfers, die sie als eine wichtige Komponente ihrer regionalen Wirtschaftsförderung ansehen. Heute gibt es drei unterschiedliche Förderinstrumente zur einzelbetrieblichen Forschungsförderung für KMU:

1. Nichtrückzahlbare Zuschüsse (in den thematisch abgegrenzten Fachprogrammen für FuE-Vorhaben einzelner Unternehmen, gemeinsame FuE-Projekte von Firmen, Verbundvorhaben von Industrie und öffentlich finanzierten Forschungseinrichtungen; in den indirekt spezifischen sowie in den Förderprogrammen für einzelne Aspekte des Innovationsprozesses);

2. Beteiligungskapital (auf Bundesebene: z. B. Förderprogramm „Beteiligungskapital für kleine Technologieunternehmen“ (BTU));

3. Innovationsdarlehen (auf Bundesebene: ERP-Investitionsprogramm).

Gegenwärtig existieren nur noch wenige Zuschussprogramme. Das Programm mit dem größten Volumen stellt dabei das FuE-Kooperationsförderprogramm für die mittelständige Wirtschaft dar. Eine zunehmende Bedeutung haben Fördermaßnahmen mit dem Instrument Beteiligungskapital und Innovationsdarlehen, die mit unterschiedlicher Intensität private Anleger integrieren. Administrativ werden diese Programme über die KfW, DAB und Technologiebeteiligungsgesellschaft (tbg) gefuihrt. Aus der scheinbar großen Vielfalt der Förderprogramme kommen für KMU mit nennenswertem Kapitalbedarf nur wenige Programme in Frage. Anhand der Struktur des Kapitalbedarfs bzw. der geplanten Verwendung der Mittel heißt das:

- Bei hohem Betriebsmittelbedarf (in erster Linie für Personal) scheiden investitions-orientierte Kreditprogramme weitgehend aus bzw. haben nur eine ergänzende Funktion. Gleiches gilt in der frühen Aufbauphase von JTU auch für solche Kreditprogramme, für die bankuibliche Sicherheiten zu stellen sind.

- Verfügen die KMU nur über eine geringe Eigenkapitalbasis bzw. Gründer nur über geringe Eigenmittel und sind in hohem Umfang FuE-Aufwendungen zu finanzieren, ist ein Finanzierungsmix erforderlich, bei dem Zuschüsse oder Beteiligungskapital eine große Rolle spielen. Gegenwärtig gibt es für Unternehmen mit Standort in Westdeutschland nur eine begrenzte Anzahl an Zuschussförderprogrammen zur Innovationsfinanzierung bzw. Angebote für öffentlich gefördertes Beteiligungskapital.

- Maßnahmen, die der Finanzierung einzelner Aktivitäten im Innovationsprozess mittelständischer Unternehmen (z.B. FuE-Kooperationen, Auftragsforschung) oder einzelner Schritte im Aufbauprozess einer Neugründung (z.B. Beratungshilfen) dienen, haben ebenfalls nur eine ergänzende Funktion, wenn ein JTU umfangreiche FuE-Arbeiten zur Schaffung eines tragfähigen Leistungsangebots durchfuihren bzw. ein innovierendes KMU sein Produktprogramm grundlegend modernisieren muss."[8]

\section{c) Spezielle Förderung der JTU}

Die Technologieförderung richtet sich an Unternehmen, bei deren Wertschöpfung die Realisierung von Innovationsprozessen eine entscheidende Rolle spielt. Da sich KMU durch eine hervorragende Forschungs- und Entwicklungstätigkeit auszeichnen, ein flexibles Marktverhalten besitzen und Hauptträger der Beschäftigung in diesem Bereich sind, kommt ihnen bei der Technologieförderung eine besondere Bedeutung zu.

In allen entwickelten Industriestaaten der Welt ist ein ausgebautes staatliches Technologieförderungssystem im wirtschaftsbezogenen FuE-Bereich gegeben.

Bis Ende der 80er Jahre herrschte in Deutschland die Zuschussfinanzierung vor. Ab Anfang der 90er Jahre traten an diese Stelle die Beteiligungsfinanzierung und die beteiligungsähnlichen Mittel zur Finanzierung der Vorlauf- und Start-up-Phase von JTU, die durch Beteiligungsgesellschaften, Kreditinstitute, Unternehmen und Privatpersonen investiert werden (vgl. Abbildung 5).

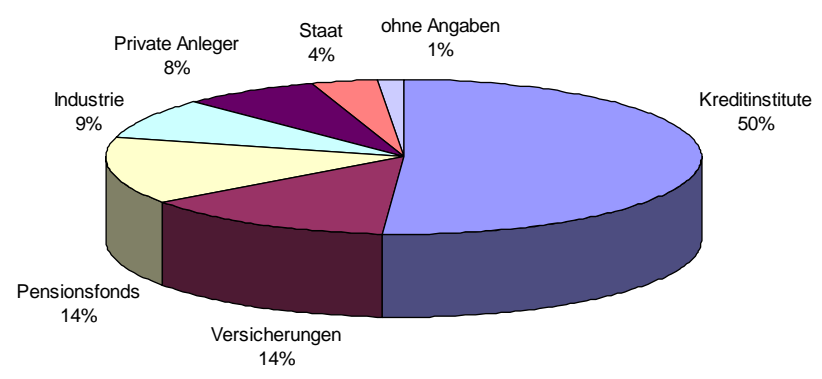

Abb. 5: Quellen des Fondsvolumens ${ }^{9}$

In diesem Zusammenhang ist der erfolgreiche Modellversuch „Beteiligungskapital fuir JTU (BJTU)“ zu nennen. Das gegenwärtige Förderprogramm „Beteiligungskapital für kleine Technologieunternehmen (BTU)“ umfasst auch mittlere Technologieunternehmen in den NBL. BTU verfolgt vorrangig nachstehende Ziele:

- nachhaltige Erhöhung des Marktangebotes für Beteiligungskapital im Bereich der Frühphasenfinanzierung der JTU und der Innovationsfinanzierung bei kleinen und mittleren Technologieunternehmen,

- Reduzierung der wesentlichen Hürden für Investments erwerbswirtschaftlicher und förderorientierter Kapitalgeber in diesem Bereich.[10] 
Das BTU-Programm zielt dabei auf Technologieunternehmen (TU), die jünger als 5 Jahre sind. In den NBL wird der Modellversuch TU im Förderprogramm „Futur“ fortgesetzt. Es zielt auf innovative technologieorientierte Unternehmen, die sich unmittelbar vor oder bereits in der Gründungsphase befinden. Dieses Programm ist mit Beratungsunterstuitzung gekoppelt. Es besteht aus nicht rückzahlbaren Zuschüssen und der Bereitstellung von Beteiligungskapital. 1998 wurde das Programm „Futur plus“ gestartet. Es reicht bis zum Jahr 2000 und stellt weitere 400 Mio. Zuschüsse und Beteiligungskapital durch die tbg zur Verfuigung. Darüber hinaus haben die meisten Bundesländer Innovations- und Existenzgründungsprogramme mit Sonderbedingungen und -konditionen für die JTU aufgelegt.

Eine weitere neuere Entwicklung der Prinzipien innovativer Finanzierungsmodelle der öffentlichen Hand zeichnet sich mit einem „Public Private Partnership“ ab. Hintergründe für diesen neuen Weg sind u. a. die knappen öffentlichen Ressourcen, der Zwang zur kostengünstigeren Leistungsverbringung, die Erschließung neuer Handlungsformen und ein neuer Ansatz der Aufgabenverteilung zwischen staatlichen Institutionen und der Privatwirtschaft hin zu einer intelligenten Kooperation.

Der Grundgedanke besteht in der effektiven Verbindung von rein staatlichen Gestaltungsaufgaben mit den Instrumenten der unternehmerischen Effizienz, dem privaten Kapital und Sachverstand. Damit soll einer wesentlichen Kostensenkung, höheren Flexibilität, spezifischen Orientierung auf eine Aufgabe bei gleichzeitiger Wahrung der Interessen des Gemeinwohls und einem verstärkten Anreiz des privaten Engagement entsprochen werden. Das Public Private Partnership könnte in sehr vielen Gebieten der Förderung von innovierenden KMU und Existenzgründungen Anwendung finden. Die Förderung sollte zur Subsidiarität der Finanzierung der KMU und JTU beitragen und sich dann zurückziehen, wenn die private Finanzierung greift.

\section{d) Kapitalbeschaffung durch Börsenemission}

Seit März 1997 besteht mit dem Start des Neuen Marktes für innovierende Unternehmen die Möglichkeit, sich Eigenkapital am organisierten Kapitalmarkt zu beschaffen. Trotz der steigenden Anzahl der gelisteten Unternehmen haben diesen Weg nur wenige innovierende Unternehmen und JTU zur Erweiterung der Eigenkapitalbasis genutzt. In Zukunft wird sich dies wahrscheinlich für die expandierenden Unternehmen ändern. Für viele JTU und innovierende Unternehmen scheint dieser Weg aber keine Finanzierungsalternative zu sein. Mit dem großen Zuwachs an Beteiligungsgesellschaften in den letzten Jahren und ihre Orientierung auf den Neuen Markt hat dieser Neue Markt indirekt die Kapitalangebote für die beteiligungskapitalsuchenden innovierenden Unternehmen und JTU's beträchtlich erweitert. Damit geht wahrscheinlich die Wirkung des Neuen Marktes über die direkte Verbesserung der Eigenkapitalbasis weniger Wachstumsunternehmen hinaus.

\subsection{Besonderheiten der Beteiligungsfinanzierung als Form der Außenfinanzierung}

Die Besonderheit der Beteiligungsfinanzierung ergibt sich daraus, dass es sowohl Eigenkapital ist, aber auch eigenkapitalähnliche Mittel umfasst, die als direkte oder stille Beteiligung bzw. als eigenkapitalähnliches Darlehen (z. B. Partiarisches Darlehen oder Darlehen mit Wandlungsrecht) außerhalb des organisierten Kapitalmarktes in Unternehmen eingebracht werden. Die Kapitaleinlage ist mit Kontroll-, Informations- und Mitentscheidungsrechten sowie zum Teil mit einer Managementunterstuitzung durch den Investor verbunden.[11]

Die Beteiligungsfinanzierung wird zum größten Teil uiber Venture Capital bzw. Venture Capital Gesellschaften (VC) realisiert. Die VC gehen in der Regel nur Minderbeteiligungen am Gesellschaftskapital der Unternehmen ein (Eigenkapitalfunktion ohne Verzinsungs- und Ruickzahlungspflicht). Die VC sind häufig nicht an einer vollen Abschöpfung der Gewinne interessiert, sondern sichern ihre Rendite über die Veräußerung ihrer Anteile nach 5 bis 10 Jahren. In der Regel unterstuitzen sie die Portfoliounternehmen aktiv mit Managementfunktionen und uiben Kontroll- und Mitspracherechte bei grundlegenden Entscheidungen aus. Eine strikte Einordnung dieser Verhältnisse ist heute schwer möglich, da viele VC ihre Bedingungen ganz konkret auf das jeweilige Portfoliounternehmen zuschneiden.

Das Beteiligungskapital wird häufig mit dem Begriff Risikokapital gleichgesetzt. Damit wird aber nur der eine Aspekt des Beteiligungskapitals betont, das Risiko. Verdeckt wird damit der zweite Aspekt, der die höheren Chancen der Kapitalgeber eröffnet. Ob in Anbetracht der Tatsache, dass von dem investierten Kapital der Mitglieder des Bundesverbands Deutscher Kapitalbeteiligungsgesellschaften (BVK) 1998 nur 2,8 \% Totalverluste ausmachen, der Aspekt des Risikos zu Recht so betont werden muss, ist eine weiter zu untersuchende Frage. Erreicht werden diese guten Ergebnisse im Beteiligungskapitalgeschäft durch die Investoren unter anderem dadurch, dass sie mit einer adäquaten innovationsorientierten Bonitätsprüifung, mit entsprechend qualifiziertem Personal und einem entsprechenden Engagement in den eingegangenen Deals die Voraussetzung dafür schaffen.

Der deutsche Beteiligungskapitalmarkt wurde 1983 etabliert und durchlebte viele Höhen und Tiefen. Seit 1997 kam es durch die Gründung neuer Fonds und die starke Aufstockung der bestehenden Fonds zu einer deutlichen Belebung des Beteiligungskapitalmarktes. Das fuihrte zu kräftigen Zuwachsraten bei neu investiertem Kapital. Im ersten Halbjahr 1999 betrug der Zuwachs 1,8 Mrd., entsprechend den Erfahrungen wird sich dieser Betrag auf 7 Mrd. bis zum Ende des Jahres 1999 erhöhen.

Der Beteiligungskapitalmarkt ist kein einheitlicher Finanzierungsmarkt. Er kann wie folgt strukturiert werden (vgl. Abbildung 6). 


\section{Beteiligungskapitalgeber}

Corporate Venture Capital Geber
Informelle Beteiligungskapitalgeber

(Business Angels)
Formelle Beteiligungskapitalgeber

Abb. 6: Struktur der Beteiligungskapitalgeber

In diese aus der Abbildung ersichtlichen Segmente des Beteiligungskapitalmarktes investieren die einzelnen Kapitalgeber je nach Interessen und Risiko- und Chanceneinschätzung.

\subsubsection{Informeller Beteiligungskapitalmarkt - Business Angel (BA)}

Das Engagement der BA in innovierende KMU und JTU ist in Deutschland ein noch relativ junges Thema, zu dem kaum empirische Informationen vorliegen. Der informelle Beteiligungskapitalmarkt gehört nicht zum organisierten Teil des Beteiligungskapitalmarktes. Das ist ein wichtiger Unterschied zum formellen Beteiligungskapitalmarkt. Informelle Investoren sind vermögende Privatpersonen, die Kapital aus ihrem Vermögen direkt und unter Umgehung der Finanzintermediäre (VC) in KMU und JTU investieren. Zwischen BA und den anderen Beteiligungskapitalgebern gibt es zunehmend Formen der Kooperation und Syndizierung, so dass der Übergang zum formellen VC-Markt häufig fließend ist.

Es gibt BA, die nur ihr Kapital zur Verfügung stellen und solche, die noch einen Mehrwert (Value Addit) einbringen. Interessant und wichtig zur Lösung der hier betrachteten Probleme sind die BA, die aktiv mit Rat und Tat und ihren Erfahrungen, Netzen sowie mit ihrem fachlichen Know-how den Unternehmen einen Mehrwert bieten können und wollen. Bei diesem Mehrwert der echten BA geht es um:

- ihr spezifisch-technisches und kaufmännisches Können und Wissen,
- ihre professionellen Kontaktnetzen,

- ihre unternehmerischen Erfahrungen (eigene Existenzgründung),

- ihre engagierte Betreuung und Beratung - Coaching.

Wegen dieser Kompetenzen sind sie in diesem Geschäft sehr wichtig. Nach Vergleichsrechnung zu dem in den USA und Großbritannien vorhandenen BA-Potenzial könnte es in Deutschland ca. 220.000 potenzielle BA und 27.000 aktive BA im o. g. Sinne geben. Durch ihre Sachkompetenz setzen die echten BA mit ihrem Engagement ein positives Signal für die Banken, denen sie ein Bonitätssignal geben (vgl. Abbildung 7). BA sind komplementär zu VC und zum Teil zu Unternehmen, die Corporate Venturing betreiben.

\subsubsection{Der Corporate Venture Kapitalmarkt}

Gegenüber den formellen Beteiligungskapitalgebern haben die Corporate Venture Capital (CVC) eine besonders hohe Kompetenz im Detailwissen der relevanten Märkte und Technologien. Die Mitarbeiter der großen Unternehmen, die beauftragt sind, die CVC zu unterstuitzen, haben viel Erfahrung in verschiedenen strategischen und operativen Fragestellungen einschließlich des Managements. Zudem können die CVC von der Reputation und dem Image der Muttergesellschaft profitieren.[13] Unter CVC kann man die Beteiligung von etablierten Unternehmungen an JTU mit Eigenkapital oder eigenkapitalähnlichen Mitteln verstehen. Im engeren Sinne ist es die Beteiligung von großen, multinationalen Unternehmen an JTU.

\section{Business Angels in Deutschland}

Viel Mut und technologisches oder Vertriebs-Know-how

Schwache (juristische) Absicherung

Häufig ohne konkrete Exit-Strategie

Aber:

Zunehmende Inanspruchnahme öffentlicher Förderung zur Risikominderung

Co-venturing mit tbg Technologie Beteiligungsgesellschaft der Deutschen Ausgleichsbank 


\begin{tabular}{|c|c|c|c|c|}
\hline & \multicolumn{4}{|c|}{ Corporate Venture Capital } \\
\hline \multirow[t]{2}{*}{$\begin{array}{l}\text { Art des CVC- } \\
\text { Investments }\end{array}$} & \multicolumn{2}{|c|}{$\begin{array}{l}\text { indirekt, externes Management } \\
\text { Investment über einen extern verwalteten, } \\
\text { eigenständigen Venture Capital Fonds }\end{array}$} & \multicolumn{2}{|c|}{$\begin{array}{l}\text { direkt, internes Management } \\
\text { direkte Minderheitsbeteiligungen des } \\
\text { kapitalgebenden Unternehmens }\end{array}$} \\
\hline & $\begin{array}{l}\text { unabhängiger Multi- } \\
\text { Investor-Fonds }\end{array}$ & $\begin{array}{l}\text { Captive Fonds des } \\
\text { kapitalgebenden } \\
\text { Unternehmen }\end{array}$ & $\begin{array}{l}\text { In-House-Fonds, } \\
\text { der durch Personal } \\
\text { des kapitalgeben- } \\
\text { den Unternehmens } \\
\text { verwaltet wird } \\
\end{array}$ & $\begin{array}{l}\text { Ad hoc/ one-off } \\
\text { Beteiligungen }\end{array}$ \\
\hline \multirow[t]{2}{*}{ Merkmale } & \multicolumn{2}{|c|}{$\begin{array}{l}\text { Fonds investiert in JTU } \\
\text { Betreuung der JTU durch VC-Management, } \\
\text { ggf. auch durch kapitalgebendes Unter- } \\
\text { nehmen, z. T. strategische Partnerschaften } \\
\text { zwischen diesem und JTU (in FuE, Produk- } \\
\text { tion, Beschaffung, Marketing, Lizensierung) }\end{array}$} & \multicolumn{2}{|c|}{$\begin{array}{l}\text { neben Finanzierung auch Manage- } \\
\text { mentunterstützung durch Personal } \\
\text { des kapitalgebenden Unternehmens, } \\
\text { strategische Partnerschaften mit dem } \\
\text { JTU } \\
\text { u. U. parallele Finanzierung der JTU } \\
\text { durch unabhängigen Venture-Capital- } \\
\text { Fonds }\end{array}$} \\
\hline & \multicolumn{4}{|c|}{$\begin{array}{l}\text { Betreuung der CVC-Engagements durch Abteilung/Personal des kapitalgebenden } \\
\text { Unternehmens oder durch eine Tochtergesellschaft. } \\
\text { u. U. integriertes Venture-Capital-Programm, das die unterschiedlichen CVC- } \\
\text { Formen mit anderen Entwicklungsprogrammen kombiniert. }\end{array}$} \\
\hline
\end{tabular}

Abb. 8: CVC-Strategien und Organisationsformen [14]

Fast alle 20 großen deutschen Unternehmen haben mittlerweile einen eigenen Venture Capital Fonds aufgelegt, dazu zählt auch die BASF in Schwarzheide und Jenoptik. Die Unternehmen unterstuitzen, beraten und fördern systematisch Mitarbeiter in den eigenen Reihen, die sich mit neuen Geschäftsideen unternehmerisch betätigen wollen. Diesen Mitarbeitern werden Freiräume, materielle Voraussetzungen und Geld beschafft, damit sie ihre innovativen Ideen umsetzen können. Insbesondere wird der Anschub unterstuitzt, um den wirtschaftlichen Erfolg vorzubereiten bzw. zu gewährleisten. Es werden aber auch zunehmend Menschen außerhalb der Unternehmen mit Ideen und Durchsetzungsvermögen, die sich als Unternehmer verwirklichen wollen, gesucht und unterstuitzt. Dies geschieht nicht selbstlos. Die großen Unternehmen verfolgen damit den Grundgedanken, dass nur mit neuen Ideen ein Unternehmen langfristig seine Ziele verwirklichen und neue Geschäftsfelder erobern kann. Gefördert werden hauptsächlich solche Ideen, die sich in einem großen Unternehmen nicht eindeutig einem Geschäftsbereich zuordnen lassen und deshalb viel Abstimmung und Koordination benötigen. Solche Verfahren lassen sich in großen Unternehmen, wenn uiberhaupt, dann nur unter großen Schwierigkeiten durchsetzen.

Angesiedelt sind die Corporate Venture Capital entweder im Haus des Großunternehmens oder auch außerhalb, wobei die o. g. Unterstützungen beibehalten werden. Die Ergebnisse der so geförderten Personen (Unternehmen) werden dann auf ihre Verwendbarkeit im Großunternehmen noch einmal speziell geprüft. Bei einer Nichtübernahme der Ergebnisse in das Unternehmen wird meistens das Ergebnis durch zu gründende Unternehmen selbst produziert und vermarktet (vgl. Abbildung 8).

\subsubsection{Formeller-institutioneller Beteiligungskapitalmarkt}

Der formelle Beteiligungskapitalmarkt wird auch häufig als institutioneller Beteiligungsmarkt bezeichnet. Dieser Bereich des Beteiligungskapitalmarktes ist bisher am stärksten entwickelt und es gibt auf diesem Gebiet sehr umfangreiche Untersuchungen und bewertete Ergebnisse. Am formellen Beteiligungskapitalmarkt sind, wie in der Abbildung 6 dargestellt, private und öffentliche Venture Capital-Gesellschaftten[15], die über Beteiligungsfinanzierungen Investitionen bei Unternehmen durchfuihren, die zumindest zum Finanzierungszeitpunkt nicht börsenreif sind. Formale Venture Capital-Gesellschaften (VCG) fuihren derartige Firmierungen typischerweise als Intermediär für Kapitalgeber durch.

Die Kapitalnehmer nehmen dabei den Charakter von Portfoliounternehmen VCG an.

Fast alle deutschen und in Deutschland tätigen ausländischen Kapitalbeteiligungsgesellschaften sind im Bundesverband Deutscher Kapitalbeteiligungsgesellschaften (BVK) organisiert.

Der Hauptsitz des seit 1998 bestehenden BVK's ist in Berlin und umfasst 115 ordentliche, sowie 35 assoziierte Mitglieder. Der BVK ist dabei nicht auf Gewinnerzielung ausgerichtet, sondern definiert seine Ziele wie folgt:

- Schaffung bestmöglicher Rahmenbedingungen für Beteiligungskapital in Deutschland,

- Erleichterung der Suche nach/des Zugangs zu Beteiligungskapital für Kapitalsuchende,

- Marktbeobachtung, Analyse von Marktentwicklungen,

- Förderung des Erfahrungsaustausches zwischen den Mitgliedern,

- Veröffentlichung von Publikationen über Beteiligungskapital in Deutschland.

Um die rasante Entwicklung des bedeutendsten deutschen Beteiligungskapitalmarktes zu verdeutlichen, werden in den folgenden Ausfuihrungen einige wichtige 
Informationen und Trends anhand der „BVK Statistik 1998“ einschließlich Begriffserklärungen vorgestellt.

\section{Ausgewählte Aspekte des aktuellen deutsche Beteili-} gungskapitalmarkt im Überblick [16]

1. Das Fondsvolumen erreichte Ende 1998 die Rekordmarke von 16,75 (13,63) Mrd. DM; gegenüiber 1997 (1996) stieg es mit 3,12 $(4,28)$ Mrd. DM um $+23 \%$ (46\%) wieder ganz beträchtlich an. Im ersten Halbjahr 1999 expandierte das Neugeschäft um weitere 1,82 Mrd. DM. Nach Prognosen des BVK wird sich dieser Wert noch auf einen neuen Rekordbetrag von rund 7 Mrd. DM erhöhen.

2. Die Höhe des Kapitals, das Ende 1998 (1997) in Beteiligungen investiert war, betrug 9,5 $(7,2) \mathrm{Mrd}$. DM, was einer Steigerung von 2,28 $(0,95)$ Mrd. DM oder $31,6(15,1 \%)$ Prozent entsprach. Es wurde in rund 3.789 (3.327) Beteiligungen gehalten.

3. Die dominierenden Quellen des Fondsvolumens waren Kreditinstitute mit 51,1\% (58,1\%), Versicherungen mit 13,8\% (11,3\%) und Pensionsfonds mit 14,2\% $(11,7 \%)$ (vgl. Abbildung 5).

4. Das Volumen neu eingegangener Beteiligungen stellte mit 3,37 (2,37) Mrd. DM einen Anstieg um 40,3\% (50\%) gegenüber 1997 (1996) dar. Die Abgänge (Desinvestments) erreichten 1998 einen Wert von 1,05 $(1,42)$ Mrd. DM. Die Anzahl neuer Beteiligungen stieg gegenuiber dem Vorjahr um knapp 48 (50) Prozent auf 1.428 (965) an.

5. Das noch nicht in Beteiligungen investierte Fondsvolumen der BVK-Mitglieder erreichte Ende 1998 den sehr hohen Wert von 7,3 (6,6) Mrd. DM. Das ist das Kapital, das in den nächsten Jahren mindestens für neue Beteiligungen bereitsteht.

6. Der wichtigste Weg im Jahr 1998 fuir die Beteiligungsgesellschaften, um eine Beteiligung zu veräußern, war der Rüickkauf durch die Alteigentuimer mit einem Anteil von 43,45\% (32,9\%). Es folgt der Verkauf an ein anderes Unternehmen mit einem Anteil von 32,3\% (52,0 \%). Einen starken Anstieg hatte der Anteil der IPOs in Deutschland zu verzeichnen. Der Anteil stieg von $4,1 \%$ auf $16,6 \%$ an.

7. Die Höhe des in den neuen Bundesländern insgesamt investierten Beteiligungskapitals spiegelt nicht den hohen Eigenkapitalbedarf der dortigen Unternehmen wider. Lediglich 1.141 (716) Mio. DM werden in 707 (615) Beteiligungen gehalten. Der Anstieg in den neuen Bundesländern betrug jedoch gegenüber dem Vorjahr 59,36\% (8,50\%) (vgl. Abbildung 9).

8. Rund $41 \%$ (57\%) (nach der Anzahl) der Bruttoinvestitionen 1998 auf dem deutschen Beteiligungskapitalmarkt floss in Branchen, die man als technologieorientiert bezeichnen kann (vgl. Abbildung 10).

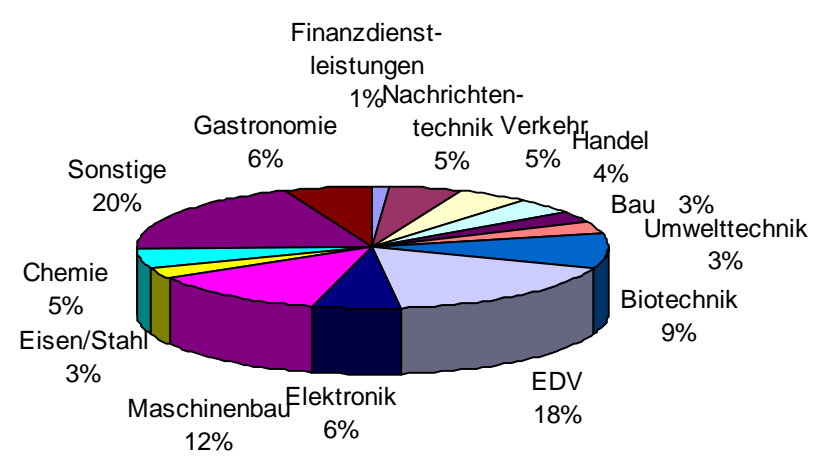

Abb. 10: Bruttoinvestitionen nach Branchen [18]

9. Das Jahr 1998 war auch für das Frühphasensegment ein Rekordjahr. Es wurden 662 (426) Frühphasenbeteiligungen über 829,11 $(371,5)$ Mio. DM neu abgeschlossen. Der durchschnittliche Beteiligungsbetrag betrug 1,61 (1,16) Mio. DM. 46 (44) Prozent (nach der Anzahl) aller neuen Engagements im deutschen Beteiligungsmarkt waren Frühphasenfinanzierungen. Damit erreichte Deutschland im innereuropäischen Ländervergleich ein beachtliches Niveau (vgl. Abbildung 11).

Komprimiert und schlaglichtartig kann der gegenwärtige Stand des Beteiligungskapitalmarktes wie folgt gesehen werden:

- Nicht nur für Deutschland waren die Jahre 1997 und 1998 erfolgreiche Jahre im Beteiligungskapitalgeschäft, sondern auch für viele andere nationale Märkte in Europa war es eine erfolgreiche Zeit.

- Beteiligungskapital stellt gegenwärtig für eine kleine, aber heterogene Gruppe von innovierenden KMU eine

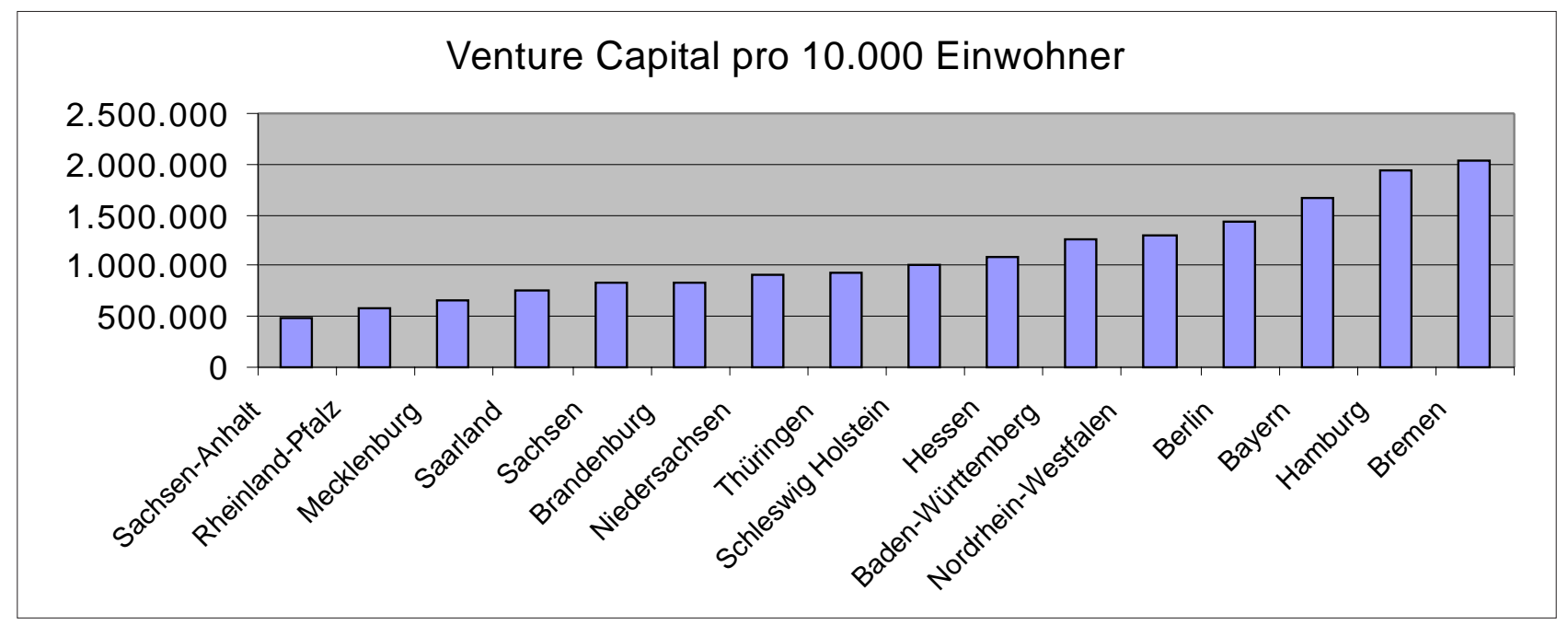

Grafik 9 - Venture Capital pro 10.000 Einwohner in Deutschland (eigene Quelle) ${ }^{17}$ 


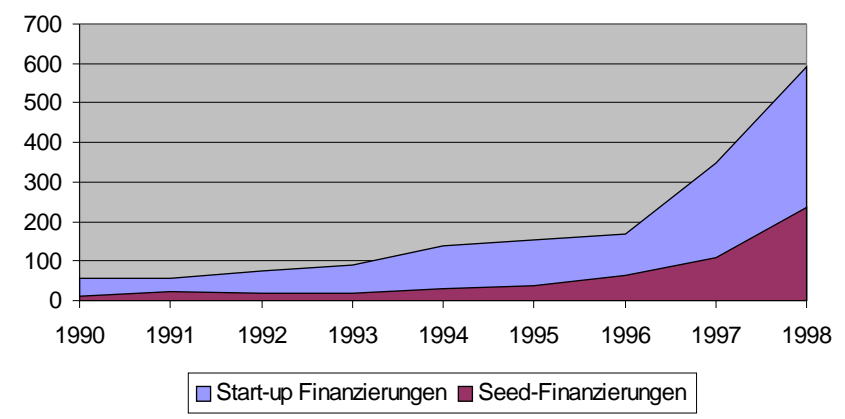

Abb. 11: Beteiligungskapital in den einzelnen Phasen [19]

interessante Finanzierung dar. Diese Finanzierung wird aber auch von Unternehmen mit ganz anderen Eigenschaften erfolgreich in Anspruch genommen.

- Gegenwärtig sind die Bedingungen fuir beteiligungskapitalsuchende innovierende KMU sehr günstig. Seitens der kapitalsuchenden Unternehmen werden die Vorbehalte gegen diese Beteiligungen oder sogar neuen Mitgesellschaftern zunehmend geringer.

- Einen merklich gestiegenen Stellenwert nimmt das Beteiligungskapital in der Finanzierung der JTU ein. Die Bereiche in Biotechnologie und Informations- und Kommunikationstechnik (primär Multimedia) nutzen diese Finanzierung besonders stark.

- Das Frühphasenengagement expandierte in den letzten Jahren besonders beeindruckend (vgl. Abbildung 4). Einen maßgeblichen Anteil an dieser Entwicklung haben die spezialisierten Seed Capital-Gesellschaften.

- Das Zusagevolumen des Förderprogramms BTU ist enorm gestiegen, $d$. h. die Förderprogramme beginnen zu greifen.

- Alle Kapitalanbieter, wie CVC, BA und formelle VC einschließlich der Seed Capital-Gesellschaften und die wertorientierten Kapitalanbieter, haben dazu beigetragen, dass heute ein großes Volumen an Kapital bereitsteht.

- Eine erfolgreiche Kapitalakquisition setzt ein professionelles Vorgehen des Kapitalnachfragers voraus. Das betrifft insbesondere die Erarbeitung eines aussagefähigen Business Planes sowie die richtige Auswahl und Verhandlungsfuihrung mit geeigneten Kapitalbeteiligungsgesellschaften.

\section{Fazit}

Die Gegebenheiten für Innovationsfinanzierungen bei KMU haben sich in den Finanzierungsquellen Fremdkapital und Selbstfinanzierung in den vergangen Jahren kaum geändert. Geändert haben sich die Bedingungen für innovierende KMU und JTU, besonders für die, die ein hohes Wachstum erwarten lassen. Denen liegt ein vielfältigerer Kapitalbeteiligungsmarkt sowie die Börsenemission auf dem Neuen Markt vor. Die öffentlichen Förderangebote haben sich für dieses Unternehmenssegment ebenfalls positiv entwickelt.

Die dargestellten Entwicklungen auf dem deutschen Beteiligungskapitalmarkt bestätigen die eingangs gemachte Aussage vom gegenwärtigen Boom auf diesem Gebiet.

\section{Anmerkungen}

[1] Mit der Wildauer Ringvorlesung „Innovationsfirmierung von KMU“" will die TFH Wildau eine Plattform für Innovations- und Erfahrungsaustausch zwischen Gründern, erfahrenen Unternehmen, kapitalsuchenden und kapitalanbietenden Unternehmen und der öffentlichen Förderung bieten und damit auch die Gründungskultur bzw. Unternehmenskultur voranbringen.

[2] vgl. Koschatzky, K.; Kulicke, M.; Nellen, O.; Pleschak, F. (Hrsg.): Finanzierung von KMU im Innovationsprozeß Akteure, Strategien, Probleme. Konferenzbeiträge, Stuttgart 1999, S. 3

[3] vgl. ebd. S. 4

[4] vgl. ebd. S. 5

[5] vgl. ebd. S. 7

[6] vgl. ebd. S. 7

[7] vgl. ebd. S. 7

[8] vgl. ebd. S. 11

[9] vgl. BVK-Statistik 1998. Berlin 1999, S. 3

[10] vgl. Koschatzky, K.; et al. (Hrsg.): Finanzierung von KMU im Innovationsprozeß, a.a.O. 1999, S. 14

[11] vgl. Frommann, H.: Venture Capital in Deutschland. Rückblick auf ein Vierteljahrhundert. In: BVK-Jahrbuch 1992. Berlin 1992, S. 31

[12] vgl. Koschatzky, K.; et al. (Hrsg.): Finanzierung von KMU im Innovationsprozeß, a.a.O. 1999, S. 100

[13] vgl. Lassat; Hemer; Eckerle; Kulicke; Licht; Nerlinger et al.: Beteiligungskapital und technologieorientierte Unternehmungen. Wiesbaden 1999, S. $148 \mathrm{ff}$.

[14] Es gibt auch andere Einteilungsmöglichkeiten der Venture Capital-Gesellschaften. Vgl. Schafczeyk, M.: Erfolgsstrategien deutscher Venture Capital-Gesellschaften. Stuttgart 1998, S. $17-20$

[15] ebd. S. $17 \mathrm{ff}$.

[16] vgl. BVK-Statistik 1997, Berlin 1998, S. 1-2, und BVK-Statistik 1998. Berlin 1999, S. 1-2

[17] vgl. BVK-Statistik 1998. Berlin 1999, S. 12

[18] vgl. ebd. S. 10

[19] vgl. ebd.

[ ] Schween, K.: Corporate Venture Capital. Wiesbaden 1996

\section{Autor}

\section{Prof. Dr. Lothar Brunsch}

Technische Fachhochschule Wildau

Fachbereich Betriebswirtschaft/Wirtschaftsinformatik

Tel. (0 33 75) 508-558

E-Mail: brunsch@wi-bw.tfh-wildau.de 